Original Research Paper

\title{
Prison Perimeter Surveillance System Using WSN
}

\author{
${ }^{1}$ Shereen Ismail, ${ }^{2}$ Eman Alkhader and ${ }^{3}$ Amal Ahmad \\ ${ }^{I}$ Department of Computer Science and Engineering, \\ American University of Ras AlKhaimah, United Arab Emirates \\ ${ }^{2}$ Department of Electrical Engineering, University of Jordan, Jordan \\ ${ }^{3}$ Department of Electrical Engineering /Computer and Communication, \\ Al-Zaytoonah University of Jordan, Jordan
}

Corresponding Author:

Miss Shereen Ismail

Department of Computer

Science and Engineering,

American University of Ras

AlKhaimah, UAE

Email: shereen.subhi@aurak.ac.ae

\begin{abstract}
The aim of this paper is to introduce a prison hybrid surveillance system using two main technologies; Wireless Sensor Network (WSN) and Unmanned Aerial Vehicles (UAVs). The system consists of three tiers; Wireless Underground Sensor Network (WUSN) in tier 0, Wireless Ground Sensor Network (WGSN) in tier 1 and Wireless Vision Sensor Network (WVSN) in tier 2 that consists of surveillance towers and Unmanned Aerial Vehicles (UAVs) equipped with multimedia sensors. Those three tiers are independent in operation and can complement one another in functionality. Such a design that utilizes the most advanced technologies proves performance in flexibility, scalability and hierarchical surveillance for a high security prison or any other military site.
\end{abstract}

Keywords: Prison Surveillance, Wireless Sensor Networks, UAV, Multicopters

\section{Introduction}

Today's life security is a very important concern in the world either for protection of critical infrastructures, highly restricted areas or even any public places. Indeed, the development of reliable and efficient security systems becomes the main interest for many researchers. For better achievement of security, advanced technologies are designed for military, police forces or security services uses. The aim of this paper is to invent a security hybrid surveillance system for perimeter patrols that can be applied around correctional facilities and/or prisons. The proposed system is mainly based on the use of Wireless Underground Sensor Network (WUSN), Wireless Ground Sensor Network (WGSN), Wireless Vision Sensor Network (WVSN) that consists of surveillance towers and a special kind of Unmanned Aerial Vehicles (UAVs); multicopters or drones equipped with high resolution multimedia sensors. The proposed system is flexible enough to be applied for security monitoring and surveillance in many other emerging sites as we will discuss later.

Basically, current WSNs consist of hundreds or thousands of intelligent tiny sensor nodes deployed uniformly or randomly over certain geographical area (Darabkh et al., 2012). Typically, these nodes deliver their sensed data collaboratively to a base station then the aggregated data to the main control operator room for further processing and data analysis.
Actually, WSNs are well suited for long-term environmental data acquisition. They have been employed recently in a large spectrum of monitoring applications such as habitat monitoring, military intrusion detection, surveillance disaster management, nuclear sites monitoring, rescue operations as well as different civilian applications (Ismail et al., 2016), because of many factors such as low cost, fast deployment, long lifetime, low maintenance and high quality of service (Ramadan and El-Rewini, 2009).

The objective in this work is to introduce a prison hybrid surveillance system consists of three tiers where WUSN is employed in tier 0 , WGSN is used in tier 1 and WVSN which consists of surveillance towers and Unmanned Aerial Vehicles (UAVs) equipped with multimedia sensors is utilized in tier 2 . Those three tiers are independent in operation and can complement one another in functionality

In WUSN, where the sensors are buried at a shallow depth underground and each one programmed with its exact location allowing the determination of any illegal movement to be easily achieved. Since their presence is hidden underground, intruders would be less likely to know about and thus take action to disable the security system (Akyildiz and Stuntebeck, 2006). Usually pressure, acoustic, or magnetic sensors are the most suitable to be used underground. In our proposed system, we employed Magnetic Induction 
(MI)-based wireless underground sensor networks as we will see in the proposed system section.

On the other hand, WGSN is considered in tier 1 of the proposed system in order to detect the intruder entering the monitored area, estimate its location and report the location information to the base station in order to be delivered directly to the main control room while the sensors keep tracking the positions' estimate; using an effective tracking algorithm; as the target moves across a certain path. Adjacent sensor nodes often monitor the same mobile intruder and collaborate to estimate its location. The sensors in tier 1 are structured based on clustering architecture with uniform grid deployment. Most of the sensors are in sleep or hibernate states most of the time to save battery energy and increase the system lifetime (Ismail et al., 2015).

Furthermore, WVSN that consists of surveillance towers and UAVs are employed in tier 2 of the proposed system. Generally, UAVs have been defined as devices used or intended to be used for flight in air that has no onboard pilot. One type of UAVs could be configured for autonomous flight with a pre-determined algorithm and called multicopters or drones (Sun et al., 2011). Remotely Piloted Vehicles (RPVs) is another type that is remotely controlled by ground operator. UAVs were primarily motivated by military applications. Afterwards, UAVs are intended to be used in a variety of civilian and commercial applications such as 3D mapping, people guidance in touristic places, rescue and searching for missing people, firefighting, police raids, agriculture and farming, photography, cars parking and public areas monitoring.

In this paper, we will discuss one of the potential applications of recently invented UAVs that serve as mobile sensors; communicate through a wireless link and equipped with cameras and any other attached sensing devices in order to detect and track the line of sight intruders in the perimeter of prisons or any protected region that need to be secured by providing visual (i.e. video and audio) information to the main control operator room.

Coordinating various technologies in such a system aims to achieve high security efficiency, scalability and long lifetime monitoring. It provides a flexible solution for prison perimeter monitoring or any other critical military sites.

\section{Brief Literature Review}

With recent advancements in the development of wireless communication and electronic devices, it is possible to fabricate efficient technologies such as sensor devices, high resolution cameras and UAVs that can be coupled to build a hybrid system in order to be used for a variety of new policing functions and extensive applications. Firstly, sensor devices have the capability of communicating with each other and the base station up to the end control operator or the administrator (Darabkh et al., 2012; Ismail et al., 2016; Ramadan and El-Rewini, 2009). Generally, WSN is a network that is formed when a set of intelligent sensor nodes are deployed randomly or manually in a physical environment to observe an event of interest. WSN monitoring includes both indoor and outdoor applications. The sensors in the vicinity of the event of interest should be able to monitor it and deliver the observed data to the base station.

Because of its wireless nature, WSNs have been employed in military, rescue, environmental, civil and healthcare applications. All these applications involve monitoring and tracking a mobile target scenario where in all of these applications the network sensors assigned the task of continuously detecting, localizing and reporting the positions of the mobile target to the base station while it's moving along a certain path.

Secondly, camera sensors have been recently developed for collecting image/video information with high resolution when used in such applications. Usually camera sensors are attached on static surveillance towers for monitoring and surveillance of interest areas such as boundary lines, airports, coastal areas and other private or public places with increased demands for protection against penetration of intruders during day or/and night time even under adverse weather conditions and over great distance.

Thirdly, multicopters or drones are special kind of UAVs that recently represent an attractive choice to be used in many emerging surveillance applications. Their small sizes simplify the take-off and retrieval while usually equipped with onboard autopilot and GPS navigation system, cameras, Wi-Fi and other different types of necessary sensors according to the intended application. The autopilot system allows it to fly autonomously to specific locations (i.e. drones) or remotely controlled by a pilot at the ground control station (i.e. RPVs). If the multicopter is equipped with image/video camera, on board camera will be placed underneath the multicopter and camera rotating mechanism and corresponding controller are necessary (Fetisov et al., 2012). Attached cameras can capture any possible intruders (vehicles or persons) and send the images or video stream back to the main processing unit in the ground control station for further processing. Afterwards, the administrator based on received information can decide and manage delivering warning alarms to the prison troops to move in case of any illegal intrusion.

In this paper, we suggest a hybrid surveillance system that utilizes these new emerging technologies. It consists of 3 tiers; WUSN to monitor the underground conditions, WGSN to detect the objects on ground, WVSN based on the use of several multicopters that 
cooperate to track any intruder within the prison perimeter area. These devices in each use certain algorithm to communicate with the main control operator in order to eventually deliver and report the final data.

The rest of the paper is organized as follows. Section 2 demonstrates our proposed system. In Section 3, the hardware implementation is illustrated. The main challenges when designing such a hybrid system is discussed and well justified in Section 4. Section 5 concludes our paper with suggested future work.

\section{Proposed System}

Many studies are presented in literature for designing secure surveillance systems for prison perimeter patrol based on various approaches. For example, locks, remote-controlled doors, pressure pads to detect footfalls, normal CCTV and many other techniques usually are used as independent layers.

In this paper, a prison hybrid surveillance system is proposed that consists of three tiers independent in operation and complement one another in functionality. Fig. 1 shows the proposed system architecture. WUGN that represents tier 0 to provide coverage under ground surface, WGSN in tier 1 to detect any moving objects above ground and surveillance towers and UAVs equipped with multimedia sensors in tier 2 , where the surveillance towers provide accurate detection as well as large detection range and UAVs provide intrusion tracking capability to track the illegal intruder after they have been detected by the tier 0 and 1 and improve the accuracy of the system through visual information in order to provide additional coverage and flexibility.

Tier 0 consists of underground vibration sensors deployed manually at a shallow depth and can communicate either with each other or with the base station in a tree based architecture.

Deployed devices must be stationary, aware of their location and synchronized to a global clock. It can detect vibration activity that indicate the availability of an intruder from a distance of 50 meter for human and around $500 \mathrm{~m}$ for a vehicle. Since their presence is hidden underground, intruders would be less likely to know about and thus take any action to disable the system.

The possible complicated underground environment that contains of soil, rocks, water and other possible substances prevents the direct use of most existing wireless solutions due to loosy channel characteristics which is related to high level of attenuation and material absorption loss, low propagation speed and limited wireless communication range (Rault et al., 2014).

New physical layer techniques in signal propagation have been recently used because of acceptable communication performance through soil or concrete medium, such as magnetic induction which makes use of magnetic antennas implemented as coils to improve the transmission range due to a much lower pathloss.
The Magnetic Induction (MI) communication (Sun et al., 2011) is a promising signal propagation technique in such a complicated medium, since the dense medium does not cause higher attenuation rate of magnetic fields than the rate in the air, the MI channel conditions do not dramatically vary as the soil properties change and MI communication can effectively transmit and receive wireless signals using a small coil of wire.

In tier 0 , because of the pre-deployment of underground sensors at varying depths and due to the main task of these sensors to detect any possible intrusion, the tree-based network architecture seems to be best suited for this purpose. We restrict the network progress to the simply connected spanning trees, since it is preferable to avoid circulating data in the network due to the limited data rate in the underground communication systems. As it is common in tree-based architecture, each sensor root node not only transmits its own sensed data, but also relays the data from the leave nodes connected to it. For this, we apply a decode-andforward relaying scheme. The data from the whole network is then guided to the base station node, which retransmits the data to the main server in control operator room for centralized processing and data analysis.

Tier 1 uses uniform grid deployment of sensors. The sensors and the base station node; as the nodes in tier 0 ; are all stationary after deployment and synchronized to a global clock. The sensors in this tier use clustering architecture. The clusters are formed dynamically according to the detection of an approaching target. The criterion in electing the $\mathrm{CH}$ is based on the distance between the detecting sensor node and the mobile target. The nearest to the target will be elected to be $\mathrm{CH}$ and order to form a cluster then observe the target. These $\mathrm{CHs}$ are autonomously connected with the base station to relay the observed data. The base station aggregates these data and sends it to the main server in the control operator room. The main server provides long-term data storage, interfaces for data access. Afterwards, the administrator may activate UAVs in tier 2 for continuous monitoring, providing live video feeds and to announce warning alarms to guide prison troops for the place of the detected intrusion if needed.

In case an intruder enters into the area, the nearest node to the target forms a cluster by inviting the surrounding nodes then keep localizing and tracking the detected target. When the target leaves the cluster vicinity, another cluster will be formed to keep tracking the target. The algorithm used in this tier was previously proposed and discussed in details in (Darabkh et al., 2012). Fig. 1 illustrates a general view of our proposed system in which for tier 1, the nodes in brown color are the sensor nodes and the nodes in red represent the currently active cluster formed during the tracking process, as mentioned previously. 


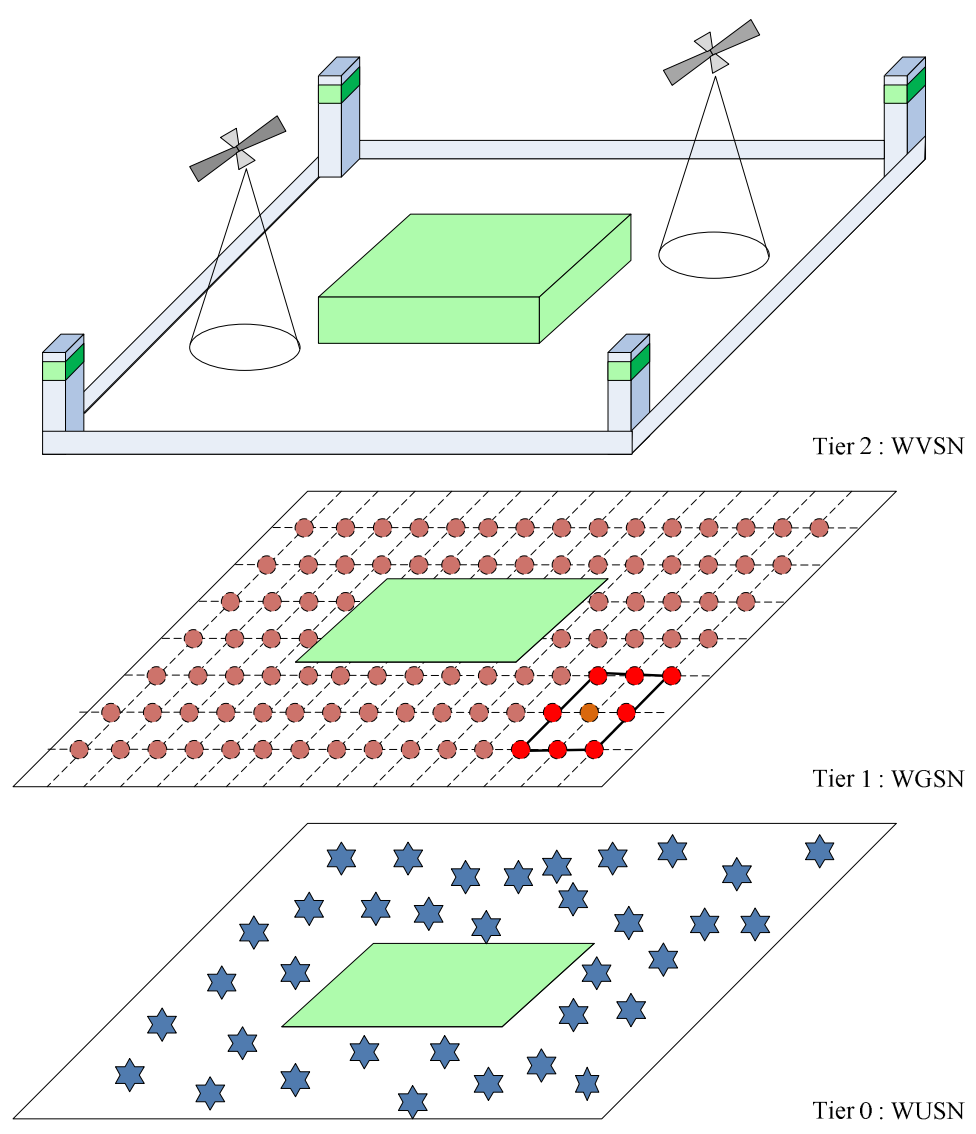

Fig. 1: the proposed prison hybrid surveillance system

Tier 0 and 1 base stations send their aggregated data to the main control operator, which, in turn, analyzes this data to take the appropriate action and activate the proper devices of tier 2 .

In tier 2, surveillance towers equipped with multimedia sensors monitor the vital signs of any possible intruders, check the perimeter area and inform the operator room in case of any intrusion. On the other hand, it may be alarmed by the control operator that there is an intrusion within its vicinity based on the received data from tier 0 and 1 nodes so it is used to activate its cameras (night vision) in order to confirm or deny detecting the intruder within that area. The use of surveillance towers can quickly find and locate the intrusion by analyzing real time video sequences and assisting the administrator in realizing more accurate detection. The multimedia sensors on the surveillance towers that detect any vital signs of intruder availability will report the detection results to the remote administrator in the control operator room. These multimedia sensors will easily and accurately help to characterize the intruder, e.g., human, vehicle or a wild animal. In case of fault alarm, the system will be returned back to the idle state. On the other hand, in case the surveillance towers receive any alarm from the control operator room, multimedia sensors near the area of interest will be directed automatically to that section of coverage according to the coordinates received within the alarm notification in order to confirm or deny the existence of any intrusion. At the same time, at least one $\mathrm{UAV}$ is dispatched according to the administrator decision in order to keep following that intruder and send continuous real time video streaming to the main control room. Consequently, prison troops and security guards can effectively move and catch the intruder.

In other cases, the surveillance towers may not be enough to locate any possible intrusion; i.e. dead zones; if the intruders will look for areas and times not properly covered by adjacent towers as Fig. 1 shows. In such a case, it is recommended to operate and lunch multi UAVs in order to achieve the required coverage. All the UAVs must be remotely connected with the control operator in a star topology for a low-latency communication.

Due to climbing capacity, reliability, ease of control, flight stability and high mobility of UAVs, the intensive human involvement in flat surveillance activities can be 
reduced. This allows valuable human resources to be allocated to decision management activities, based on the received information from such devices. However, harsh weather conditions; such as clouds, fog or dust; can also impinge on the surveillance capability of UAVs.

Moreover, as we stated earlier, UAVs could be configured with predetermined algorithm or preprogrammed with certain GPS coordinates or flight plans to move regularly in $3 \mathrm{D}$ within the perimeter space so can autonomously patrol the area in case of any expected security threat. Any detected intruder will be tracked using measurements from multiple UAVs (i.e. cameras) that do not have direct communication between each other and only communicate directly with the UAV pilot at the ground control station.

On the other hand, as each sensor in tier 0 and tier 1 is programmed with its location information as it is deployed, the exact location of any illegal crossing is easily determined in the first place in collaboration. These sensors are intended to keep tracking the detected intruder until the area of the event can be covered by the nearest surveillance tower then number of UAVs is activated and take off for continuous visual tracking.

\section{Hardware Implementation}

In this section, we are going to describe the main features and basic functionalities of the different hardware components of the proposed system.

It is noteworthy to state that the combination of WUSN, WGSN, surveillance towers and UAVs in such a hybrid system will provide additional input and unattended data sources to achieve the required coverage.

In tier 1, ultrasonic motion sensors have been installed. Indeed, such sensors; under some conditions; mayprone to false alarms another type of detection system may be required to compliment the ultrasound system.

Since skilled intruders know how to pass through space without making highly audible sounds, in this system we suggest to use ultrasonic sensors in conjunction with passive infrared sensors to increase sensitivity especially at critical crossing points (i.e., near entrance and egress points). Newer models combine both technologies within one system.

The type of UAVs in tier 2 is highly dependent on the perimeter area in which for example, multirotor do not need a lot of space for starting or landing (both automatically possible). While quadrocopter (4 motors/propellers) using a 10 Ah battery and efficient motors that will be able to fly 30-45 min; depending on the payload which represent the onboard autopilot and GPS navigation, cameras, Wi-Fi and any other types of sensors with approximate speed ranges from 5 to $10 \mathrm{~m} / \mathrm{s}$. If we assume the speed is $5 \mathrm{~m} / \mathrm{s}$, this means approximately $12 \mathrm{~km}$ of flight distance can be crossed horizontally. On the other hand, fixed wing UAV system will probably be able to fly a distance of $50 \mathrm{~km}$ with the same battery storage. But it requires much more space for starting and landing. Silent UAVs are best suited for such an application. To achieve the required silence, UAV should be 30 meter on average above groundsurface to prevent the unmanned vehicle from being heard.

Attached to the surveillance towers, the camera sensors that play an important role to provide visual information in order to determine the intrusion identity. We assume that a thermo cameras for night time operation and a VIS camera for daylight operation are the best suited for such a system.

\section{Conclusion and Future Work}

Recently, the new technologies; such as WSN and UAVs; have been employed in many critical applications (Felemban, 2013.) like:

- Environmental monitoring: for example, forest detection, habitat monitoring and animal tracking, flood detection, intelligent irrigation, forecasting and weather prediction

- Commercial applications for example, seismic activities prediction and monitoring

- Military applications: for example, enemy tracking, borders patrol and security purposes

- Health applications, such as Tracking and monitoring of patients and doctors

- Transport systems such as monitoring of traffic, dynamic routing management and car parking management

- Industrial applications: for example, automated systems monitoring

Due to the rapid concern on employing new technologies to achieve high security systems, we proposed in this paper a hybrid system using various emerging technologies for prison surveillance and perimeter patrol. The system consists three independent tiers: WUSN in tier 0 that uses tree based architecture, WGSN in tier 1 that is based on clustering architecture while we use surveillance towers and UAV technology in tier 2 equipped with multimedia sensors to achieve the maximum coverage.

As a future work, other parameters can be considered to study the system performance out of which, the effect of intruder speed on such a system, the rate of false alarm especially for tier 0 and 1 and merging new surveillance techniques in such a hybrid system to achieve the maximum security and the required coverage.

For such a design, many challenges may rise such as communication and hardware reliability as the case with WUSN in tier 0 , the real time video streaming 
synchronization and autofollow setup on UAVs in tier 2 to keep tracking the intruder object will it is moving, and system accuracy and security especially when various technologies have been used for such a hybrid system.

\section{Acknowledgement}

Special thanks to the American University of Ras Al Khaimah for providing incentive fund for this research work and for covering the fees of its publication.

\section{Author's Contributions}

Shereen Ismail: She conceived and planned the presented idea, supervised the findings of this work, and coordinating the work among co-authors.

Eman Alkhader: She contributed to the data gathering task and collaborated with the first author in designing the idea.

Amal Ahmad: She was responsible for collocting the experimental data. Also, she participated in the writing and proofreading of this manuscript.

\section{Disclosures}

There is no conflict of interests regarding the publication of this paper. We also confirm that funding in the "Acknowledgment" section did not lead to any conflict of interests regarding the publication of this manuscript.

\section{References}

Akyildiz, I.F. and E.P. Stuntebeck, 2006. Wireless underground sensor networks: Research challenges. Ad Hoc Networks, 4: 669-686.

Darabkh, K.A., S.S. Ismail, M. Al-Shurman, I.F. Jafar and E. Alkhader et al., 2012. Performance evaluation of selective and adaptive heads clustering algorithms over wireless sensor networks. J. Network Comput. Applications, 35: 2068-2080. DOI: $10.1016 /$ j.jnca.2012.08.008

Felemban, E., 2013. Advanced border intrusion detection and surveillance using wireless sensor network technology. Int. J. Communications Network System Sci., 6: 251-259.

DOI: $10.4236 /$ ijens.2013.65028

Fetisov, V., O. Dmitriyev, L. Neugodnikova, S. Bersenyov and I. Sakayev, 2012. Continuous monitoring of terrestrial objects by means of duty group of multicopters. Ufa State Aviation Technical University, Ufa, Russia,

Ismail, S., E. Alkhader and S. Elnaffar, 2016. Object Tracking in wireless sensor networks: Challenges and solutions. J. Comput. Sci., 12: 201.212.

DOI: $10.3844 /$ jcssp.2016.201.212
Ismail, S.S., E.I. Al Khader and K.A. Darabkh, 2015. Static clustering for target tracking in wireless sensor networks. Global J. Technol.

Ramadan, R. and H. El-Rewini, 2009. Deployment of sensing devices: A survey. Southern Methodist University, USA.

Rault, T., A. Bouabdallah and Y. Challal, 2014. Energy efficiency in wireless sensor networks: A top-down survey. Computer Netw., 67: 104-122. DOI: 10.1016/j.comnet.2014.03.027

Sun, Z., P. Wang, M.C. Vuran, M.A. Al-Rodhaan and A.M. Al-Dhelaan et al., 2011. MISE-PIPE: Magnetic induction-based wireless sensor networks for underground pipeline monitoring. Ad Hoc Netw., 9: 218-227. DOI: 10.1016/j.adhoc.2010.10.006

Sun, Z., P. Wanga, M.C. Vuran, M.A. Al-Rodhaan and A.M. Al-Dhelaan et al., 2011. BorderSense: Border patrol through advanced wireless sensor networks. Ad Hoc Netw., 9: 468-477. DOI: $10.1016 /$ j.adhoc.2010.09.008 